\title{
Diabetes mellitus und Schwangerschaft
}

\section{Typ-1- und Typ-2-Diabetes, Gestationsdiabetes}

\author{
Diabetes mellitus and pregnancy \\ Type 1 and type 2 diabetes, gestional diabetes
}

Helmut Kleinwechter, Ute Schäfer-Graf

\begin{tabular}{|l|lll|r|}
\hline Übersicht & & & \\
Lernziele & 55 & & Untersuchungen zur fetalen & \\
Gesundheitsziele & 55 & & Zustandsdiagnostik & 64 \\
Epidemiologie & 55 & & Behandlung geburtshilflicher & \\
Typ-1- und Typ-2-Diabetes & 56 & & Komplikationen & 65 \\
Gestationsdiabetes & $\mathbf{6 0}$ & & \\
\hline
\end{tabular}

\section{Lernziele}

Dieser CME-Beitrag soll die wichtigsten Aspekte zur Betreuung eines präkonzeptionell bekannten Typ-1oder Typ-2-Diabetes erläutern. Außerdem werden Screening, Diagnostik, Therapie und Nachsorge bei Gestationsdiabetes (GDM) beschrieben. Grundlage sind die Leitlinienfassungen der Deutsche Diabetes-Gesellschaft (DDG) und der Deutschen Gesellschaft für Gynäkologie und Geburtshilfe (DGGG) (evidenzbasierte S3-Leitlinien, Praxisversionen, Patientinnenversionen [1-6]) sowie neuere Publikationen.

\section{Gesundheitsziele}

Die WHO-Europa und die International Diabetes Federation (IDF) haben 1989 in der St.Vincent-Deklaration als 5. Hauptziel für den Zeitraum von 5 Jahren formuliert, dass Verlauf und Ergebnis von Schwangerschaften bei Frauen mit Diabetes an die Resultate von Frauen ohne Diabetes angeglichen werden sollen. Die WHOEuropa hat außerdem 1999 in ihrem Programm „GESUNDHEIT21“ (Gesundheit für alle im 21.Jahrhundert) die Gesundheitsziele konkretisiert und für diabetische Schwangerschaften eine Verbesserung der Ergebnisse um ein Drittel bis zum Jahr 2020 vorgeschlagen.

\section{Epidemiologie}

Schwangerschaften bei präkonzeptionell bekanntem Typ-1- und Typ-2-Diabetes und GDM bedürfen einer gemeinsamen Betreuung durch spezialisierte Diabetologen, Geburtsmediziner und Neonatologen in enger Kooperation mit Hebammen, Augenärzten und anderen Fachgebieten. Im Jahr 2011 wurde in deutschen Geburtskliniken im Rahmen der Perinatalstatistik bei 640000 Schwangerschaften in $1 \%$ der zur Geburt betreuten Schwangeren ein präkonzeptionell bekannter Diabetes registriert ( $=6500)$. Damit hat sich die Prävalenz in den letzten 10 Jahren verdoppelt. Der Anteil des Typ-2-Diabetes nimmt zu und wird in Deutschland auf 20-30\% geschätzt (Abb. 1 und Abb. 2).

Schwangerschaften bei präkonzeptionellem Diabetes und Gestationsdiabetes erfordern eine interdisziplinäre Betreuung durch spezialisierte Diabetologen, Geburtsmediziner und Neonatologen. 


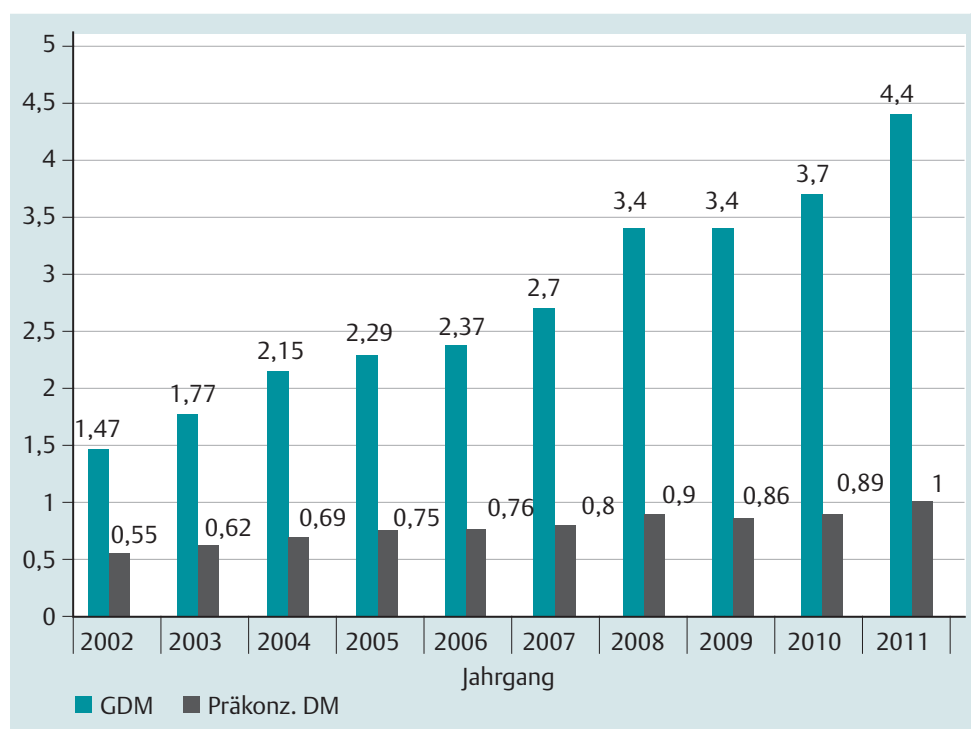

Abb.1 Relative Häufigkeiten von präkonzeptionellem Diabetes und Gestationsdiabetes (GDM) in Deutschland 2002 - 2011, erfasst zum Zeitpunkt der Geburt durch die Perinatalstatistiken.

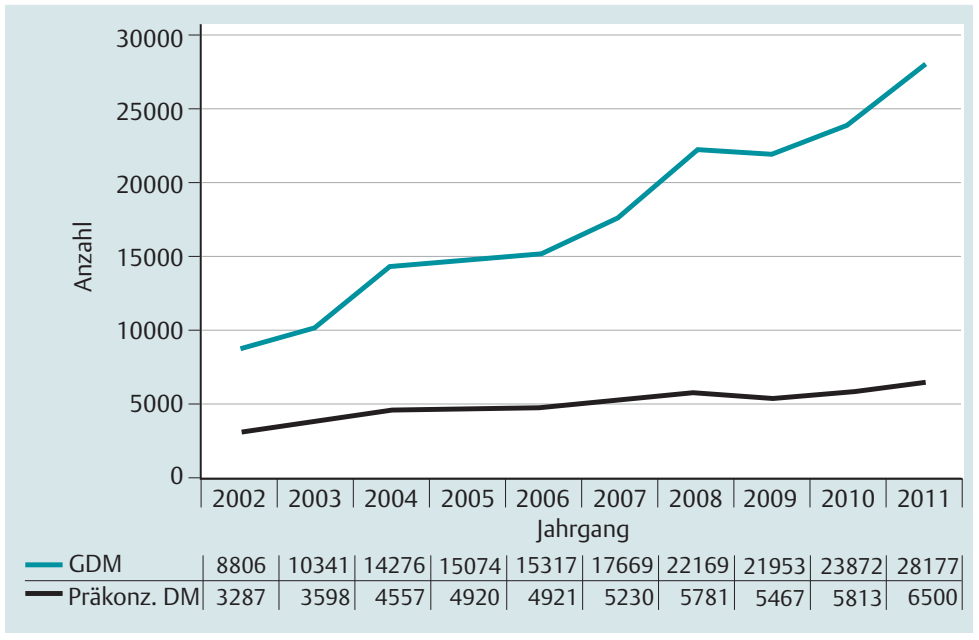

Abb.2 Absolute Häufigkeiten von präkonzeptionellem Diabetes und Gestationsdiabetes (GDM) in Deutschland 2002 - 2011, erfasst zum Zeitpunkt der Geburt durch die Perinatalstatistiken.
- Wirksamkeit bei hoher Sicherheit (Pearl-Index)

- geringe Nebenwirkungen

- nutzerinnenfreundlich (den Wünschen der Frau und ihres Partners entsprechend)

- Einflüsse auf den Kohlenhydrat- und Fettstoffwechsel

- Einflüsse auf diabetesspezifische Folgekomplikationen

Besonders sorgfältig müssen Frauen mit einer Erkrankungsdauer über 10 Jahre und mikrovaskulären Komplikationen mit evtl. zusätzlichem Nikotinkonsum beraten werden. In manchen Fällen wird man zwischen den sozialen Folgen einer ungewollten Schwangerschaft und erhöhten Gesundheitsrisiken durch sichere Kontrazeption abwägen müssen.

Frauen, die bereits länger als 10 Jahre an einem Diabetes erkrankt sind und bei denen mikrovaskuläre Komplikationen oder ein Nikotinabusus vorliegen, müssen hinsichtlich der Kontrazeption besonders sorgfältig beraten werden.

Stoffwechselziele. Präkonzeptionell soll eine normnahe Stoffwechseleinstellung mit einem $\mathrm{HbA}_{1 \mathrm{c}}<7 \%$ erzielt werden. Besser ist eine Einstellung des $\mathrm{HbA}_{1 \mathrm{c}}$ $<6,5 \%$, wenn dies ohne gehäufte schwere Hypoglykämien möglich ist. Die Blutglukose-Zielwerte (kapillär mit dem Handmessgerät der Schwangeren gemessen) nach Eintritt der Schwangerschaft sind in Tab. 1 aufgeführt.

Mittlere Blutglukosewerte eines Tages, bestehend aus sechs Werten (vor den Hauptmahlzeiten und eine Stunde danach) von $<90 \mathrm{mg} / \mathrm{dl}(5,0 \mathrm{mmol} / \mathrm{l})$ deuten auf eine zu straffe Einstellung mit dem Risiko fetaler Wachstumsretardierung hin, mittlere Blutglukosewerte $>110 \mathrm{mg} / \mathrm{dl}(6,1 \mathrm{mmol} / \mathrm{l})$ gelten als nicht ausreichend gut eingestellt. Die postprandialen Werte (vorzugsweise nach einer Stunde) sind für die fetale Prognose von vorrangiger Bedeutung, hiernach sind die nachfolgenden präprandialen Insulindosierungen zu adaptieren. Eine sofortige postprandiale Korrektur oberhalb der angegebenen Zielwerte erhöht das Hypoglykämie-Risiko durch Überlagerung der Insulinwirkprofile, deshalb soll diese erst ab $200 \mathrm{mg} / \mathrm{dl}$ $(11,0 \mathrm{mmol} / \mathrm{l})$ vorgenommen werden.

Kontrazeption. Ziel der präkonzeptionellen Vorbereitung ist eine geplante Schwangerschaft unter optimierten Voraussetzungen. Daher ist eine sichere Kontrazeption bis zum Erreichen dieser Voraussetzungen erforderlich [7]. Die geeignete Kontrazeption wird individuell ausgewählt. Folgende Faktoren werden bei der Auswahl der Methode berücksichtigt:
Der $\mathrm{HbA}_{1 \mathrm{c}}$-Wert (IFCC-Standard) sollte während der Schwangerschaft alle 4-6 Wochen bestimmt werden und im Referenzbereich für Gesunde liegen. Die aktuell gemessenen Blutglukosewerte sind für die Stoffwechselführung des Diabetes entscheidend. 


\section{Tabelle 1}

Zielwerte für die Blutglukose-Selbstkontrolle (Messung mit Handmessgerät).

\begin{tabular}{|c|c|c|}
\hline \multirow[t]{2}{*}{ Zeit } & \multicolumn{2}{|c|}{ Plasma-Äquivalent } \\
\hline & $\mathrm{mg} / \mathrm{dl}$ & $\mathrm{mmol} / \mathrm{l}$ \\
\hline nüchtern, präprandial & $65-95$ & $3,6-5,3$ \\
\hline $1 \mathrm{~h}$ postprandial & $<140$ & $<7,8$ \\
\hline $\begin{array}{l}\text { mittlere Blutglukose } \\
\text { Mit Messungen } 1 \mathrm{~h} \text { postprandial }\end{array}$ & $90-110$ & $5,0-6,1$ \\
\hline nachts 2-4 Uhr & $>65$ & $>3,6$ \\
\hline
\end{tabular}

Präkonzeptionell sollte der $\mathrm{HbA}_{1 \mathrm{c}}$ auf $<7 \%$ oder besser noch auf $<6,5 \%$ - wenn dies ohne häufige Hypoglykämien möglich ist - eingestellt werden.

Humangenetische Beratung. Das Risiko der Kinder einer Mutter mit Typ-1-Diabetes, an einem Typ-1-Diabetes zu erkranken, liegt bei 5,3\% nach 20 Jahren. Ist auch der Vater an Typ-1-Diabetes erkrankt, beträgt das 5-Jahres-Risiko $11 \%$, hat neben der Mutter auch ein Geschwister Typ-1-Diabetes, liegt die Rate nach 5 Jahren bei $12 \%$. Das Risiko in der Allgemeinbevölkerung beträgt bis zum 25 . Lebensjahr ca. $0,3 \%$.

Abort- und Fehlbildungsrisiko. Das Risiko für Frühaborte ist erhöht und abhängig von der präkonzeptionellen Stoffwechseleinstellung: Sehr niedrige $(<5 \%)$, aber auch sehr hohe $\mathrm{HbA}_{1 \mathrm{c}}$-Niveaus $(>8,5 \%)$ erhöhen das Abortrisiko. Numerische Chromosomenanomalien (z.B. Trisomie 21) treten bei diabetischen Schwangerschaften nicht gehäuft auf.

Das Risiko für große Fehlbildungen liegt mit 8,8\% im Mittel rund 4-fach höher als in der Allgemeinbevölkerung. Dies gilt für Typ-1- und Typ-2-Diabetes gleichermaßen. In geplanten Schwangerschaften ist das Fehlbildungsrisiko, bedingt durch die gezielte Beratung, die bessere Stoffwechseleinstellung und BlutglukoseSelbstkontrollen geringer als in ungeplanten. Fehlbildungen betreffen vorwiegend das Herz und herznahe Gefäße (Risiko: 4-fach), Neuralrohrdefekte (Risiko: 2 - bis 3-fach) und multiple Fehlbildungen. Ein Diabetes-spezifischer Fehlbildungstyp existiert nicht.

Das Risiko für Frühaborte und für Fehlbildungen ist abhängig von der präkonzeptionellen Stoffwechsellage erhöht.
Reproduktionsmedizin bei diabetischen Frauen. Bei unerfülltem Kinderwunsch gibt es - nach Abschluss der reproduktionsmedizinischen Diagnostik in einem dafür ausgestatteten Zentrum - für die infrage kommenden Methoden der Sterilitätsbehandlung keine Einschränkungen. Zyklusstörungen normalisieren sich häufig allein durch eine optimierte Insulintherapie.

Reproduktionsmedizinische Diagnostik und Sterilitätsbehandlung unterliegen bei Frauen mit Diabetes keinen Einschränkungen.

Einnahme von Folsäure und Jod. Allen Schwangeren mit Kinderwunsch wird eine Substitution mit 400 $800 \mu \mathrm{g}$ Folsäure/Tag empfohlen, beginnend mindestens 4-8 Wochen präkonzeptionell (z. B. beim Absetzen der Kontrazeption) bis zum Abschluss von Schwangerschaftswoche (SSW) 12 zur Prävention von Neuralrohrfehlbildungen. Zur ausreichenden Versorgung des Fetus mit Jod ist eine medikamentöse Jodprophylaxe mit mindestens $200 \mu \mathrm{g}$ Jodid/Tag zu empfehlen.

4-8 Wochen präkonzeptionell sollten $400-800 \mu \mathrm{g}$ Folsäure/Tag zur Prävention von Neuralrohdefekten substituiert werden.

Pharmakotherapie. Insulin ist bei Schwangerschaften mit manifestem Diabetes die Therapie der Wahl. Schwangere mit Typ-2-Diabetes sollen daher von oralen Antidiabetika auf Insulin umgestellt werden. Die intensivierte konventionelle Insulintherapie (ICT) oder bei entsprechender Indikation die kontinuierliche subkutane Insulininfusion (CSII) mittels Insulinpumpe sind die Insulinstrategien der Wahl und vom Ergebnis her gleichwertig.

Klinisch wichtige Indikationen zur Pumpentherapie ergeben sich schon bei Planung der Schwangerschaft:

- rezidivierende schwere Hypoglykämien

- langfristig deutlich erhöhte $\mathrm{HbA}_{1 c}$-Verläufe

- Dawn-Phänomen

- inhärent labiler Stoffwechsel („brittle diabetes“)

- Wunsch der Patientin (Präferenzentscheidung)

Die Pumpentherapie sollte möglichst 3 Monate vor der geplanten Konzeption begonnen werden. Die ICT verlangt während der Schwangerschaft in manchen Fällen besondere, auf den Einzelfall zugeschnittene Kunstgriffe, um die Insulinwirkung bei niedrigeren Zielwerten zu optimieren und zu stabilisieren. Beispiele hierfür sind: 
- Zusätzlich NPH-Insulin (Basalinsulin) mittags und zum Abendessen

- Verschieben der Nachtbasis-Injektion auf 23-24 Uhr (statt $22 \mathrm{Uhr}$ )

- Injektion kurzwirksamen Insulins morgens gegen 5 Uhr (zum Abfangen des Blutglukoseanstiegs vor dem Frühstück)

- Ausreichender Bolus-Essabstand von 15-30 Minuten, besonders vor den Hauptmahlzeiten

- Beschränkung der Menge der Kohlenhydrat-Einheiten pro Mahlzeit, wichtig beim Frühstück (maximal 3 Kohlenhydrateinheiten)

- Mengenbeschränkung oder Meiden freien Zuckers (z.B. Süßigkeiten, Haushaltszucker)

Limitiert wird die straff normale Einstellung durch das mütterliche Hypoglykämie-Risiko. Daher müssen individuelle Zielvereinbarungen bei gehäuften schweren Hypoglykämien, eingeschränkter HypoglykämieWahrnehmung, besonderen Lebensumständen und problematischem sozialen Umfeld getroffen werden. Mit schweren Hypoglykämien ist bei Typ-2-Diabetes kaum zu rechnen.

Der Insulinbedarf steigt im zweiten Schwangerschaftsdrittel beginnend kontinuierlich an (+50-100\% bis zur Geburt) und kann besonders bei adipösen Schwangeren mit Typ-2-Diabetes sehr hoch sein. Nach 20 SSW sinkt bei Typ-1-Diabetes das Hypoglykämie-Risiko trotz der steigenden Insulin-Dosierungen. 2-4 Wochen vor der Geburt fällt der Insulinbedarf z.T. deutlich ab. Mit Beginn der Geburt reduziert sich der basale Insulinbedarf um ca. 50\% (z. B. unter CSII). Peripartal (im Kreißsaal) wird nur kurzwirksames Insulin, je nach lokalen Gepflogenheiten auch intravenös, verabreicht. Postpartal wird die Insulinsubstitution innerhalb weniger Tage individuell neu angepasst, als Orientierung dient der präkonzeptionelle Bedarf. Die werdende Mutter sollte unbedingt einen Insulinplan mit ihren präkonzeptionellen Insulindosierungen zur Geburt mitbringen.

Insulinstrategien der Wahl sind die intensivierte konventionelle Insulintherapie und bei entsprechender Indikation die kontinuierliche subkutane Insulininfusion mittels Insulinpumpe.

Wahl des Insulinpräparates. Humaninsulin ist das Medikament der Wahl. Die Fortsetzung der Therapie mit den Insulinanaloga Detemir und Aspart ist unbedenklich [8]. Da bei CSII für kurzwirksame Insulinanaloga vs. Humaninsulin außerhalb der Schwangerschaft ein signifikanter Vorteil bei schweren Hypo- glykämien belegt werden konnte, kann dieser Befund in eine Einzelfallentscheidung auch für die Schwangerschaft einbezogen werden.

Medikament der Wahl ist Humaninsulin. Die Insulinanaloga Detemir und Aspart können weiter verwendet werden.

\section{Diabetologische Notfälle}

Hypoglykämie. Schwere Hypoglykämien der Schwangeren mit Notwendigkeit einer Glukose- oder Glukagoninjektion müssen vermieden werden. Sie gefährden die Mutter, akute Gefahren für den Fetus bestehen nicht. Durch eine stabile, normnahe Einstellung können außerdem hypoglykämische Warnzeichen wie Zittern, Schwitzen oder Tachykardie zunehmend unterdrückt sein und schließlich durch unzureichende hormonelle Gegenregulation ganz fehlen. Schwere Hypoglykämien treten vorrangig bei Typ-1-Diabetes in den ersten 20 SSW und dann überwiegend während des Schlafes auf [9]. Der Partner oder ein anderer Angehöriger muss über Hypoglykämien informiert und in die Anwendung des Glukagon-Notfallsets eingewiesen sein. Risiken für schwere Hypoglykämien sind:

- schwere Hypoglykämien im Jahr vor der Schwangerschaft

- eingeschränkte Hypoglykämie-Wahrnehmung

- Diabetesdauer $>10$ Jahre

- $\mathrm{HbA} 1 \mathrm{c}<6,5 \%$

- Blutglukose vor dem Schlafen < $108 \mathrm{mg} / \mathrm{dl}(6 \mathrm{mmol} / \mathrm{l})$

Schwere Hypoglykämien gefährden die Mutter und müssen vermieden werden.

Ketoazidose. Die Ketoazidose bei diabetischer Schwangerschaft ist eine kritische Notfallsituation und kann selbst bei normalen Blutglukosewerten auftreten [10]. Der ketoazidotische Notfall kann sich in wenigen Stunden aufbauen und kommt bei Typ-1- und auch Typ-2Diabetes vor. Klinisches Leitsymptom sind Bauchschmerzen. Die sofortige stationäre Einweisung in notärztlicher Begleitung in eine geeignete Klinik ist angezeigt. Hier soll unmittelbar die Komatherapie nach hausinternem Behandlungsschema begonnen und das weitere Vorgehen in enger Absprache zwischen Diabetologen, Geburtsmedizinern und Neonatologen erfolgen. Klinische Risiken für Ketoazidosen sind:

- Erbrechen

- Infektionen (z. B. Pyelonephritis)

- Non-Adhärenz (z.B. häufiges Weglassen von Mahlzeiten, fragmentarische Selbstkontrolle ohne schrift- 
liches Protokoll, Vergessen von Insulininjektionen, mangelnde psychosoziale Unterstützung)

- unzureichende Stoffwechseleinstellung

- Ablehnen oder Ambivalenz gegenüber der Schwangerschaft

- gestörtes Essverhalten

- Katheterdiskonnektion oder Insulinleck anderer Ursache bei Pumpentherapie

- Gabe von $\beta$-Mimetika zur Wehenhemmung

- Glukokortikoide zur Induktion der fetalen Lungenreife

Die Ketoazidose ist eine kritische Notfallsituation bei Schwangeren und kann bei Typ-1- und Typ-2Diabetes vorkommen. Leitsymptom ist Bauchschmerz.

\section{Komplikationen und Begleiterkrankungen}

Arterielle Hypertonie. Zu unterscheiden sind Hochdruckformen mit bereits präkonzeptioneller Therapie (Ziel-RR < 140/90 mmHg) und schwangerschaftsspezifische Hochdruckerkrankungen (Gestationshypertonie, Präeklampsie), die erst nach 20 SSW manifest werden und erst ab 160/100 mmHg behandelt werden. Die Therapieführung der schwangerschaftsspezifischen Hochdrucktherapie nach 20 SSW liegt beim Geburtsmediziner.

ACE-Hemmer, AT1-Blocker und Aliskiren sollen bereits präkonzeptionell umgestellt werden, um ein fetales RAS-Blockade-Syndrom zu vermeiden [11]. AlphaMethyl-Dopa ist das Medikament der Wahl, eine Kombination mit Metoprolol ist möglich. Diuretika sollen in der Schwangerschaft nicht neu angesetzt, eine schon präkonzeptionell begonnene Diuretikatherapie kann fortgesetzt werden.

Präkonzeptionell und bis 20 SSW liegt der Zielblutdruck bei $<140 / 90 \mathrm{mmHg}$. Bei erhöhten Werten nach der 20. SSW erfolgt eine Therapie erst ab 160/100 mmHg. ACE-Hemmer, AT1-Blocker und Aliskiren sind in der Schwangerschaft kontraindiziert.

Diabetische Retinopathie. Sie ist die häufigste mikrovaskuläre Komplikation bei diabetischen Schwangeren und kann erstmals während der Schwangerschaft manifest werden. Bereits vorhandene Läsionen können sich während der Schwangerschaft verschlechtern, meist im 3. Trimenon. Progressionsrisiken sind:
- Schwangerschaft per se (hormonelle Umstellung)

- ungünstiger präkonzeptioneller Netzhautbefund

- unzureichende präkonzeptionelle Laserkoagulation

- arterielle Hypertonie

- diabetische Nephropathie

- Rauchen

- hohes perikonzeptionelles $\mathrm{HbA}_{1 \mathrm{c}}$-Niveau

- Diabetesdauer >10 Jahre

- Anämie

Augenärztliche Untersuchungen sind immer mit erweiterten Pupillen angezeigt: Sofort nach Diagnose der Schwangerschaft und danach alle 3 Monate bis zur Geburt, bei auffälligen Befunden auch öfter. Zur Pupillenerweiterung können die üblichen Parasympatholytika verwendet werden. Der Augenarzt soll Netzhautspezialist sein.

Augenärztliche Untersuchungen mit erweiterten Pupillen sind sofort nach Diagnose der Schwangerschaft und danach alle 3 Monate bis zur Geburt angezeigt.

Diabetische Nephropathie. Eine Schwangerschaft per se führt nicht zu einer Abnahme der glomerulären Filtrationsrate (GFR) nach der Geburt. Nach Diagnose der Schwangerschaft ist zu Beginn jedes Trimenons ein Albumin-Screening zu empfehlen (Mittelwert aus zwei spontanen Urinproben zu beliebiger Tageszeit). Es korreliert bei Schwangeren gut mit der Albuminurie im $24 \mathrm{~h}$-Sammelurin und zeigt bei erstmaligem Auftreten u.a. ein erhöhtes Präeklampsie- und Frühgeburtsrisiko an. Wegen der Möglichkeit falsch negativer und falsch positiver Befunde bei einer Bestimmung von Albumin ist die gleichzeitige Messung des Urinkreatinins zu empfehlen und das Ergebnis als Urin-Kreatinin-Ratio zu dokumentieren [Tab.2].

Die Nephropathie-Stadien 1a/1b sind mit einer erhöhten Präeklampsie- und Frühgeburtenrate assoziiert. Dieses Risiko wird durch eine Blutdruckeinstellung auf Zielwerte $<140 / 90 \mathrm{mmHg}$ reduziert. Die Frühgeburtenrate vor der 34.SSW kann außerdem durch Behandlung bei normotensiven Schwangeren mit Typ-1Diabetes und Mikroalbuminurie mit $\alpha$-Methyl-Dopa reduziert werden [12].

Nephropathien ab Stadium 3 nach KDOQI bei diabetischer Schwangerschaft sind selten, aber mit hohen fetalen, neonatalen und kindlichen Risiken assoziiert (Frühgeburtenrate, Wachstumsretardierungen, intrauteriner Fruchttod, perinatale/neonatale Mortalität, psychomotorische Retardierungen im Kindesalter). 
Tabelle 2

Mögliche Bestimmungen der Urin-Albumin-Konzentration in der Schwangerschaft.

\begin{tabular}{llll}
\hline $\begin{array}{l}\text { Methode } \\
\text { 24h-Urin }\end{array}$ & Normoalbuminurie & Mikoralbuminurie & Makroalbuminurie \\
\hline $\begin{array}{l}\text { Spontanurin: } \\
\text { Albumin-Kreatinin-Ratio }\end{array}$ & $30-300 \mathrm{mg} / 24 \mathrm{~h}$ & $>300 \mathrm{mg} / 24 \mathrm{~h}$ & $\begin{array}{l}\text { Bewertung } \\
\text { Sammlung }\end{array}$ \\
\hline Spontanurin & $<30 \mathrm{mg} / \mathrm{g}$ Kreatinin & $30-300 \mathrm{mg} / \mathrm{g}$ Kreatinin & $>300 \mathrm{mg} / \mathrm{g}$ Kreatinin $\quad$ Alternative: Mittelwert aus zwei \\
\hline
\end{tabular}

Außerdem besteht nach der Geburt ein erhebliches Risiko für die Mutter: Aufnahme in das Dialyseprogramm, erhöhte Mortalität noch vor Erreichen des Erwachsenenalters ihrer Kinder. Schwangerschaften bei diabetischen Frauen während Hämo- oder Peritonealdialyse sowie nach Nieren- oder Nieren-Pankreas-Doppeltransplantation wurden zwar beschrieben, sind aber sehr selten und mit weiteren Problemen verbunden.

Bei diabetischer Nephropathie und Kinderwunsch soll auf besonders hohe mütterliche und fetale Risiken hingewiesen werden bei

- einem Serumkreatininwert ab 1,5 mg/dl

(ab $133 \mu \mathrm{mol} / \mathrm{l}$ ),

- einer Nephropathie ab Stadium 3 (Kreatinin-

Clearance $<60 \mathrm{ml} / \mathrm{min} / 1,73 \mathrm{~m}^{2}$ ),

- schwer einstellbarer arterieller Hypertonie.

Eine individuelle Risikobeurteilung in Zusammenarbeit mit dem Nephrologen ist unverzichtbar. Die Beurteilung des Einzelfall-Risikos kann bedeuten, dass von einer Schwangerschaft abgeraten werden muss.

Zu Beginn jedes Trimenons ist ein AlbuminScreening zu empfehlen. Nephropathien ab Stadium 3 sind selten, gehen aber mit einem hohen fetalen, neonatalen und kindlichen Risiko einher.

Diabetische Neuropathie. Bei geplanter Schwangerschaft und einer Diabetesdauer > 10 Jahre soll geprüft werden, ob es Hinweise für eine diabetische Gastroparese, eine orthostatische Hypotonie oder Hypoglykämie-Wahrnehmungsstörungen gibt. Eine Schwangerschaft per se führt nicht zum Neuauftreten oder zur Progression neuropathischer Veränderungen.

Makroangiopathie. Frauen mit Diabetes haben ein erhöhtes kardiovaskuläres Risiko, welches durch die Schwangerschaft u.U. weiter gesteigert wird. Gefährdet sind Frauen mit langer Diabetesdauer, höherem Lebensalter, Nephropathie, arterieller Hypertonie und Raucherinnen. Frauen nach Myokardinfarkt sollen mindestens ein Jahr mit einer Schwangerschaft warten. Vorrangig ist eine individuelle Risikoanalyse in Zusammenarbeit mit dem Kardiologen. Bei erheblich eingeschränkter linksventrikulärer Pumpfunktion wird man von einer Schwangerschaft abraten müssen.

Schilddrüse. Bei Frauen mit Diabetes besteht eine hohe Prävalenz für eine Autoimmunthyreoiditis. Präkonzeptionell oder bei Schwangerschaftdiagnose soll ein Screening mittels TSH-Bestimmung erfolgen. Bei erhöhtem TSH-Wert schließen sich die Bestimmung von fT4 und TPO-AK sowie eine Schilddrüsensonografie an. Subklinische Hypothyreosen müssen mit L-Thyroxin substituiert werden (Ziel-TSH $<2,5 \mu \mathrm{U} / \mathrm{ml}$ ).

\section{Besonderheiten bei Typ-2-Diabetes}

Schwangerschaften bei Typ-2-Diabetes nehmen zu. Die perinatalen Risiken sind genauso hoch wie bei Typ-1Diabetes. Schwangere mit Typ-2-Diabetes haben im Vergleich zu Typ-1-Diabetes eine höhere AdipositasRate, ein höheres Lebensalter, eine kürzere Diabetesdauer und eine höhere Rate an Begleitrisiken und -medikation. Sie gehören häufiger einer ethnischen Minorität an. Außerdem liegt häufiger eine chronisch arterielle Hypertonie vor, Präeklampsien sind andererseits seltener.

Die präkonzeptionelle Vorbereitung ist schlechter, und die Frauen nehmen seltener Folsäure perikonzeptionell ein. Die Erstvorstellung erfolgt meistens erst nach Abschluss der Embryogenese, und ein hoher Prozentsatz wird noch in der Frühschwangerschaft mit oralen Antidiabetika behandelt. Präkonzeptionell befindet sich ein hoher Anteil von Schwangeren mit Typ-2Diabetes nicht in diabetologischer Betreuung, und bei 
ca. $30 \%$ war 6 Monate vor der Konzeption kein $\mathrm{HbA}_{1 \mathrm{c}}$ dokumentiert. Der Anteil ungeplanter Schwangerschaften ist höher als bei Typ-1-Diabetes. Bei dieser Gruppe von Schwangeren ist daher die Zusammenarbeit mit den betreuenden Hausärzten besonders wichtig.

Typ-2-Diabetes darf bei Schwangeren nicht verharmlost werden. Die Risiken sind dem Typ-1-Diabetes vergleichbar. Eine präkonzeptionelle Schulung und Umstellung von oralen Antidiabetika auf Insulin sowie eine diabetologische Mitbetreuung sind notwendig. Die Stoffwechselziele entsprechen denen des Typ-1-Diabetes. Eine restriktive Handhabung der Gewichtszunahme bis zur Geburt ist von Vorteil (Ziel: $<5 \mathrm{~kg}$ ) [13].

Patientinnen mit Typ-2-Diabetes und Kinderwunsch sollten präkonzeptionell eine Schulung und Umstellung von oralen Antidiabetika auf Insulin erhalten.

\section{Gestationsdiabetes}

\section{Definition und Epdemiologie}

Gestationsdiabetes (GDM) ist definiert als eine Glukosetoleranzstörung, die entweder in der Frühschwangerschaft durch eine erhöhte Nüchtern-Plasmaglukose oder im Zeitraum $24+0$ bis $27+6$ SSW mit einem oralen 75g-Glukosetoleranztest (oGTT) unter Standardbedingungen und mit qualitätsgesicherter Glukosemessung aus venösem Plasma diagnostiziert wird (Tab.3). Damit schließt ein manifester Diabetes mellitus einen GDMaus. In $1-2 \%$ der Fälle wird eine Glukokinase-Genmutation (MODY-2, autosomal-dominante Vererbung) durch eine Schwangerschaft demaskiert. Im Jahr 2011 betrug die GDM-Prävalenz in Deutschland 4,4\%, entsprechend 24000 Fälle.

Gestationsdiabetes ist definitionsgemäß eine Glukosetoleranzstörung, die erstmals während der Schwangerschaft mit einem standardisierten $75 \mathrm{~g}$ oralen Glukosetoleranztest diagnostiziert wird.

\section{Pathophysiologie}

Die mütterliche Hyperglykämie induziert eine fetale Hyperinsulinämie mit funktionellen und morphologischen Kurzzeit- und Langzeitfolgen. Nach den Erkenntnissen der HAPO-Studie besteht zwischen jedem der Blutglukosewerte nüchtern, nach 1 und 2 Stunden ein

\section{Tabelle 3}

Grenzwerte zur Diagnose des Gestationsdiabetes im venösen Plasma nach IADPSG-Konsensus-Empfehlungen. Ein erhöhter Wert reicht zur Diagnose eines Gestationsdiabetes aus.

\begin{tabular}{|llc|}
\hline Zeitpunkt $\mathbf{2 4 + \mathbf { 0 }} \mathbf{- 2 7 + 6} \mathbf{~ S S W}$ & \multicolumn{2}{c|}{ Grenzwerte venöses Plasma } \\
\hline mg/dl & $\mathbf{m m o l} / \mathbf{I}$ \\
\hline nüchtern & 92 & 5,1 \\
\hline nach einer Stunde & 180 & 10,0 \\
\hline nach zwei Stunden & 153 & 8,5 \\
\hline
\end{tabular}

kontinuierlicher Zusammenhang mit perinatalen Ergebnisparametern, ohne dass ein Schwellenwert erkennbar ist [14]. Beim GDM handelt es sich um eine metabolisch komplexe Störung mit Einflüssen aus Substraten des Kohlenhydrat-, Lipid- und Eiweißstoffwechsels, bei der traditionell aber nur die Glukose gemessen wird.

\section{Diagnostik vor 24 SSW}

In der frühen Schwangerschaft wird bei Frauen mit Risiken (Tab.4) ein manifester Diabetes mellitus ausgeschlossen. Hierzu wird nur eine Nüchternglukose oder eine Gelegenheitsglukose gemessen und bei auffälligem Ergebnis wiederholt. Nach dem Flussdiagramm kann auch zu diesem Zeitpunkt ein GDM diagnostiziert werden (Abb.3).

\section{Screening und Diagnostik mit $24+0$ bis $27+6$ SSW}

In diesem Zeitraum wird zur Diagnostik ein 75g-oGTT von 2 Stunden Dauer unter Standardbedingungen durchgeführt. Die Mutterschaftsrichtlinie schreibt seit 2012 vor, einen 50g-Glukose-Suchtest vor dem Diagnosetest einzusetzen. Ein Screening-Wert nach einer Stunde von $135 \mathrm{mg} / \mathrm{dl}$ (7,8 mmol/l) oder höher gilt als positives Screening, ein Wert ab $201 \mathrm{mg} / \mathrm{dl}$ (11,2 mmol/l) bereits als GDM. Die Blutglukosemessung aus venösem Plasma muss richtliniengemäß qualitätsgesichert erfolgen (Tab.5). Zusätzlich soll jeder Arzt, der verantwortlich diagnostische Blutglukose-Einzelmessungen durchführt, Präzisionsmaße erheben und freiwillig an Ringversuchen teilnehmen. Handmessgeräte messen nur kapilläres Blut und sind deshalb ungeeignet. 


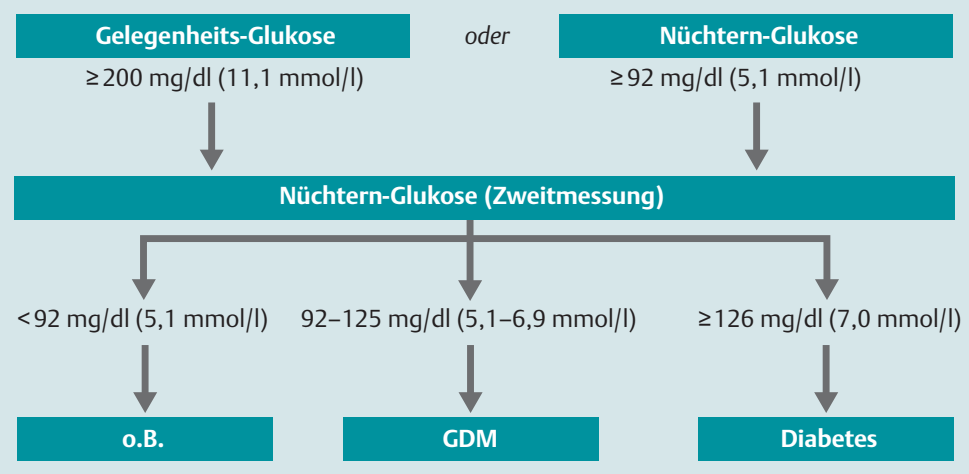

Abb. 3 Flussdiagramm Nüchtern-Glukosekategorien nach erhöhter Gelegenheits-Glukose oder erhöhter Nüchtern-Glukose vor der 24. SSW. Bei erhöhter Gelegenheits-Glukose kann mit einer Zweitmessung der Gelegenheits-Glukose $\geq 200 \mathrm{mg} / \mathrm{dl}$ ( $11,1 \mathrm{mmol} / \mathrm{l})$ die Diagnose eines manifesten Diabetes bestätigt werden.

Gemessen wird entweder direkt aus venösem Plasma nach Versand ins Labor oder mit einem für die Diagnostik zugelassenen Point-of-care-testing-Gerät (POCT-Gerät) aus venösem Vollblut mit Umrechnung in venöses Plasma (Faktor: 1,11), es sei denn, das Gerät ist bereits plasmakalibriert. Für den Laborversand sollen Entnahmegefäße mit Zusatz von NaF+Citrat verwendet werden, um die Glykolyse sofort zu hemmen. Bei Sofortmessung ist nur der Zusatz eines Gerinnungshemmers erforderlich (z.B. EDTA). Die diagnostischen Grenzen zeigt Tab. 3.

Vor dem Diagnostetest (75 g oGTT) muss mit $24+0-27+6$ SSW ein 50g-Glukose-Suchtest durchgeführt werden. Handmessgeräte zur Blutglukoseselbstkontrolle sind für Screening und Diagnostik ungeeignet.

\section{Erstinformation und Beratung}

Nach Diagnosestellung des GDM soll die Schwangere zunächst in einem ausführlichen ärztlichen Gespräch in angstabbauender Atmosphäre mit der Problematik und den geplanten therapeutischen Schritten vertraut gemacht werden. Sie wird im Gegensatz zum präkonzeptionellen Diabetes erstmals mit dem Begriff „Diabetes“ konfrontiert. Dieses Gespräch ist nicht an Assistenzpersonal oder Hebammen delegierbar.

\section{Therapie}

Blutglukoseselbstkontrolle ist Teil der Therapie und ermöglicht eine objektivierende Reflektion der Schwangeren auf ihr Ess- und Trinkverhalten und ihren Einsatz körperlicher Bewegung. Für die ersten 1-2

\section{Tabelle 4}

Risikoanalyse auf manifesten Diabetes bei Erstvorstellung in der Schwangerschaft. GDM= Gestationsdiabetes. IGT = impaired glucose tolerance; IFG = impaired testing glucose.

Alter $\geq 45$ Jahre

$\mathrm{BMI} \leq 30 \mathrm{~kg} / \mathrm{m}^{2}$ präkonzeptionell

körperliche Inaktivität

Eltern oder Geschwister mit Diabetes

Angehörige einer ethnischen Risikopopulation

(z. B. Asiatinnen, Lateinamerikanerinnen)

Geburt eines Kindes $\geq 4500 \mathrm{~g}$

GDM in der Vorgeschichte

arterielle Hypertonie (Blutdruck $\geq 140 / 90 \mathrm{mmHg}$ ) oder Einnahme von Medikamenten zur Therapie der arteriellen Hypertonie

Dyslipidämie präkonzeptionell (HDL $<35 \mathrm{mg} / \mathrm{dl}[0,9 \mathrm{mmol} / \mathrm{l}]$ und/oder Triglyceride $>250 \mathrm{mg} / \mathrm{dl}[2,82 \mathrm{mmol} / \mathrm{l}])$

polyzystisches Ovarsyndrom

Prädiabetes (IGT/IFG/ $/ \mathrm{HbA}_{1 \mathrm{c}} \geq 5,7 \%$ ) bei früherem Test (unabhängig von früherem GDM)

andere klinische Zustände, die mit Insulinresistenz assoziiert sind (z. B. Acanthosis nigricans)

Vorgeschichte mit KHK, pAVK, zerebral-arterieller Durchblutungsstörung

Einnahme kontrainsulinärer Medikation (z. B. Glukokortikoide)

Wochen misst die Schwangere täglich 4 Werte, vor dem Frühstück und jeweils 1 Stunde nach Beginn der Hauptmahlzeiten. Sind die Werte im Zielbereich, dann reicht ein Wert pro Tag im Rotationsverfahren aus.

Medizinische Ernährungstherapie. Ernährungstherapie heißt, dass die Schwangere ihr Verhalten ändert und diese Änderung überprüft wird. Am Anfang steht die strukturierte, individuelle Ernährungsanamnese durch eine Fachkraft, dann lernt die Schwangere auf der Basis ihrer Ess- und Trinkgewohnheiten die Quantifizierung der Kohlenhydrate nach Kohlenhydrat-Einheiten in einer Schulung. Der Kalorienbedarf orientiert sich an der körperlichen und beruflichen Belastung und 
kann bei Adipositas deutlich vermindert werden, ca. $1600 \mathrm{kcal} /$ Tag sollten aber nicht unterschritten werden. Gewichtszielkorridore nach den IOM-Leitlinien werden vorgegeben, $z$. B. für adipöse Schwangere $5-9 \mathrm{~kg}$.

Bewegung, Sport. Regelmäßige körperliche Bewegung oder Sport verbessern die Belastbarkeit während Schwangerschaft und Geburt. Die Rate an Insulintherapien und die Insulintagesdosis werden reduziert. Sport kann während der Schwangerschaft fortgesetzt werden, bezüglich der Risiken von bestimmten Sportarten mit Gelenkbelastung (z.B. Tennis, Badminton) und hohem Verletzungsrisiko (z.B. Kampfsportarten) sowie schwangerschaftsspezifischen Kontraindikationen (z.B. vorzeitige Wehen, Blutungen) soll rechtzeitig durch die Frauenärztin individuell beraten werden. Auch während der Schwangerschaft kann bei Wunsch der Schwangeren mit einem aeroben Ausdauertraining oder Kraftübungen auf leichtem bis mittlerem Niveau neu begonnen werden.

Pharmakotherapie. Können die Stoffwechselziele nicht erreicht werden, wird mit einer Insulintherapie begonnen. Dies ist in etwa $20 \%$ der Fälle erforderlich. Zur Indikationsstellung der Insulintherapie soll neben den Blutglukoseergebnissen das Wachstumsprofil des Fetus im Ultraschall herangezogen werden [15]. Ein Ultraschallbefund soll innerhalb von 2 Wochen nach GDMDiagnose vorliegen.

Sind mehr als 50\% der Blutglukosewerte innerhalb der ersten 2 Wochen erhöht, soll die Indikation für Insulin geprüft werden, auch wenn ein Ultraschallergebnis noch fehlt. Auffällig sind auch wiederholt gemessene Nüchternwerte $>110 \mathrm{mg} / \mathrm{dl}(6,1 \mathrm{mmol} / \mathrm{l})$ und Werte $>162 \mathrm{mg} / \mathrm{dl}$ (9,0 mmol/l) zu irgendeinem Zeitpunkt.

Eine Modifikation der Blutglukoseziele wird in Abhängigkeit vom Ultraschallbefund vorgenommen:

1. Bei asymmetrischer Makrosomie des Fetus mit einem Abdominalumfang $>75$. Perzentile werden die Blutglukoseziele nüchtern um $10 \mathrm{mg} / \mathrm{dl}$ $(0,55 \mathrm{mmol} / \mathrm{l})$ und postprandial um $20 \mathrm{mg} / \mathrm{dl}$ $(1,1 \mathrm{mmol} / \mathrm{l})$ niedriger gesetzt.

2. Bei gesicherter fetaler Wachstumretardierung werden die Ziele entsprechend um $10 \mathrm{mg} / \mathrm{dl}$ (0,55 mmol/l) bzw. $20 \mathrm{mg} / \mathrm{dl}(1,1 \mathrm{mmol} / \mathrm{l})$ höher gesetzt.

Orale Antidiabetika und GLP-1-Analoga sind mangels Zulassung nicht indiziert, auch nicht Metformin. Die Pharmakotherapie wird mit Humaninsulin in Form

\section{Tabelle 5}

Empfohlene Schritte der diagnostischen Blutglukose-Qualitätskontrolle, adaptiert nach RiliBÄK Februar 2008.

\begin{tabular}{|c|c|}
\hline $\begin{array}{l}\text { Kontroll- } \\
\text { schritt }\end{array}$ & Maßnahme \\
\hline 1 & $\begin{array}{l}\text { verwendetes Blutglukose-Messsystem nach Anweisungen des Her- } \\
\text { stellers zur Qualitätskontrolle bedienen, Ergebnisse dokumentieren }\end{array}$ \\
\hline 2 & $\begin{array}{l}\text { benutzungstäglich Gerät mit einem physikalischen und/oder elektro- } \\
\text { nischen Standard kontrollieren (wird vom Hersteller mitgeliefert) }\end{array}$ \\
\hline 3 & $\begin{array}{l}\text { an jedem Tag, an dem Patientenmessungen durchgeführt werden, } \\
\text { Kontrollprobeneinzelmessung durchführen und beurteilen - Toleranz } \\
\pm 11 \%\end{array}$ \\
\hline 4 & $\begin{array}{l}\text { überschreitet ein Kontrollprobeneinzelmesswert die Fehlergrenze, ist } \\
\text { das Messverfahren für weitere Patientenmessungen gesperrt }\end{array}$ \\
\hline 5 & $\begin{array}{l}\text { nach der Abweichung suchen, Ursache beseitigen, verantwortliche } \\
\text { Person entscheidet Freigabe nach klinischer Relevanz, Vorgang doku- } \\
\text { mentieren }\end{array}$ \\
\hline 6 & $\begin{array}{l}\text { nach Abschluss eines Kontrollzyklus Berechnung des relativen qua- } \\
\text { dratischen Mittelwertes der Messabweichung vom Kontrollproben- } \\
\text { Zielwert-Toleranz } \pm 11\end{array}$ \\
\hline 7 & freiwillige Teilnahme am Ringversuch jedes Quartal-Toleranz $\pm 15 \%$ \\
\hline
\end{tabular}

einer intensivierten Insulintherapie umgesetzt und mit einer Tagesdosis von 0,3-0,5 IE Insulin/kg aktuellem Körpergewicht begonnen. Unterdosierungen sollen vermieden werden. Kurzwirksame Insulinanaloga sind nur dann angezeigt, wenn mit kurzwirksamen Humaninsulinen die postprandialen Spitzen innerhalb von 1-2 Wochen nicht zielgerecht eingestellt werden können.

Adipöse Schwangere sollten maximal 5-9kg Gewicht zunehmen. Regelmäßige körperliche Bewegung und Sport reduzieren die Rate an Insulintherapien und die Insulintagesdosis. Können die Stoffwechselziele mit einer Ernährungstherapie nicht erreicht werden, muss eine Insulintherapie begonnen werden.

\section{Nachsorge}

Unabhängig davon, ob gestillt wird oder nicht, ist ein standardisierter oGTT 6-12 Wochen nach der Geburt bei der Mutter angezeigt. Aktuelle Befunde aus Deutschland zeigen im Mittel 3 Monate nach der Geburt bei den Müttern eine Häufigkeit von 6\% mani- 
festem Diabetes und bei weiteren 15\% eine gestörte Glukosetoleranz, ganz überwiegend IGT. Frauen mit einer IGT nach GDM sind eine ideale Zielgruppe für Präventionsmaßnahmen - sie haben noch eine lange Lebensspanne vor sich und sind für mindestens 20 Jahre für Erziehung, Schule, Ausbildung und Entlassen ihrer Kinder in die Selbstständigkeit verantwortlich. Die Nachsorge kann einen Beitrag dazu leisten, dass sie diese Lebensphase bei uneingeschränkter Gesundheit und vermindertem kardiovaskulärem Risiko gestalten kann.

Das kumulative Diabetesrisiko nach GDM liegt 8 Jahre nach GDM in Deutschland bei 53\% [16]. Ist der erste oGTT nach der Schwangerschaft unauffällig, sollte alle 2-3 Jahre erneut ein oGTT durchgeführt werden. Liegen Risiken vor, dann ist ein oGTT jährlich zu empfehlen. Zu den frühzeitig fördernden Faktoren der Diabetes-Manifestation zählen:

- GDM-Diagnose vor 24 SSW

- Präkonzeptioneller BMI $\geq 30 \mathrm{~kg} / \mathrm{m}^{2}$

- Nüchtern-Blutglukosewert im oGTT $\geq 105$ mg/dl

- Insulintherapie

- positive Inselzellautoantikörper

- ethnische Risikogruppe (vorrangig Asien, Lateinamerika)

- Stilldauer<3 Monate oder primäres Abstillen

Ein besonders hohes Diabetesrisiko haben Frauen mit positiven Autoantikörpern gegen Inselzellen zum Zeitpunkt der Geburt - 8 Jahre nach GDM sind sie zu 97\% manifest diabetisch und zu $81 \%$ bereits nach einem Jahr. Sie bedürfen einer engmaschigen Überwachung. Zielgruppe für eine Autoantikörperbestimmung (AntiGAD und Anti-IA2, falls beide negativ auch Anti-ZnT8) sind Frauen mit einer Insulintherapie und einem BMI $<30 \mathrm{~kg} / \mathrm{m}^{2}$. Die Autoantikörperbestimmung wird bei Beginn der Insulintherapie in der Schwangerschaft empfohlen.

Frauen haben bei GDM eine erhöhte Erkrankungsrate an einer peripartalen Depression. Als Suchinstrument ist der in deutscher Sprache validierte Fragebogen „Edinburgh Postnatal Depression Scale“ (EPDS) geeignet. Ein Score von>10 weist auf eine depressive Verstimmung hin, die fachspezifisch weiter abgeklärt werden sollte.

Kinder von Müttern mit GDM haben erhöhte Risiken für Übergewicht/Adipositas sowie IGT und Diabetes schon im Kindes- und Jugendalter, die Inzidenz ist abhängig von der Höhe der Glukosewerte der Mutter im oGTT während ihrer Schwangerschaft. Übergewich- tige Neugeborene von Müttern mit GDM mit europäischer Herkunft hatten zu 50\% mit 11 Jahren ein metabolisches Syndrom. Kinderärzte sollen daher über den GDM der Mutter informiert werden und entsprechend der kindlichen Entwicklung Parameter des kardiovaskulären Risikos erheben (BMI, Blutdruck, Blutglukose/ oGTT, Lipidwerte).

Ist ein erster standardisierter oGTT 6-12 Wochen nach der Geburt bei der Mutter mit Gestationsdiabetes negativ, sollte dieser alle 2 - 3 Jahre wiederholt werden.

\section{Untersuchungen zur fetalen Zustandsdiagnostik}

Zeitpunkt und Intensität der geburtshilflichen Überwachung sind abhängig vom Diabetestyp, von vorhandenen Folgekomplikationen des Diabetes und vom Verlauf der Erkrankung während der Schwangerschaft.

\section{Ultraschalluntersuchungen}

Die Mutterschaftsrichtlinien sehen drei Ultraschalluntersuchungen vor, die bei diabetischen Schwangeren durch Zusatzuntersuchungen zu ergänzen sind:

- 8-12 SSW: körperliche Integrität der Schwangerschaft und Herzaktionen

- 11 - 14 SSW: Nackentransparenz-Messung (optional)

- 19-22 SSW: differenzierte Organdiagnostik (mindestens Level DEGUM II)

- ab 24 SSW: alle 2-4 Wochen Biometrie, bei Auffälligkeiten auch häufiger

- Geburtsgewichts-Schätzung am Termin

Sonografische Hinweiszeichen für eine diabetesspezifische Makrosomie sind:

- Zunahme des Abdomenumfangs > 75. Perzentile (nach Hadlock) bei normalem Wachstum des Kopfumfangs

- Abdomenumfang bezogen auf die SSW erheblich größer als der Kopfumfang (dysproportionales Wachstum), Hinweis: Kopf-Abdomen-Ratio <0,9-1,0

Dopplersonografie. Die Indikation für diese Untersuchung wird unabhängig von der Diabetesdiagnose der Schwangeren gestellt. Bei Wachstumsretardierung des Feten stellt sie eine zusätzliche Überwachungsmethode dar. 
Sonografische Hinweiszeichen für eine diabetesspezifische Makrosomie sind eine Zunahme des Abdomenumfangs $>75$. Perzentil und ein dysproportionales Wachstum des Abdomenumfangs (Kopf-Abdomen-Ratio $<0,9-1,0)$.

\section{Kardiotokografie (CTG)}

Die Häufigkeit der CTG-Kontrollen soll an die individuellen fetalen und mütterlichen Risiken angepasst werden. Im Allgemeinen werden CTG-Ableitungen bei insulinbehandelten Schwangeren ab 32 SSW begonnen und häufiger eingesetzt, je näher die Schwangerschaft den errechneten Entbindungstermin erreicht. Hierdurch kann, besonders bei fetalen Risiken wie Wachstumsretardierung oder makrosomen Tendenzen, eine drohende intrauterine Asphyxie rechtzeitig erfasst werden. Bei diätetisch behandeltem GDM wird mit dem CTG ab errechnetem Entbindungstermin begonnen.

\section{Behandlung geburtshilflicher Komplikationen}

\section{Infektionen}

Die Häufigkeit von Infektionen des Urogenitaltraktes bei diabetischen Schwangeren ist erhöht und mit gesteigerter Frühgeburtenrate assoziiert. Daher werden regelmäßige Kontrollen und ggf. eine frühzeitige antibiotische Therapie empfohlen.

\section{Frühgeburtsbestrebungen}

Wehenhemmung (Tokolyse). Vorzeitige Wehen werden mit dem Ziel einer Verlängerung der Schwangerschaft bis zum Abschluss einer Lungenreifeinduktion behandelt. Auf eine Tokolyse über einen längeren Zeitraum soll verzichtet werden. Die orale Tokolyse mit einem $\beta_{2}$-Mimetikum oder Magnesium ist ineffektiv. Die i.v.-Tokolyse mit einem $\beta_{2}$-Mimetikum (Fenoterol) führt wegen der Bettruhe und häufig gleichzeitigen Glukokortikoidgabe zum erheblichen Anstieg der Blutglukosewerte, sodass die Insulintherapie kurzfristig angepasst werden muss. Der Oxytocin-Antagonist Atosiban ist stoffwechselneutral und als i.v.-Tokolytikum der ersten Wahl anzusehen. Primär wird heute zunächst p.o. Nifedipin („Off Label Use“) eingesetzt.
Lungenreifeinduktion. Das Frühgeburtenrisiko (Geburt vor vollendeter 37.SSW) ist bei präkonzeptionellem Diabetes im Mittel fast 5-fach erhöht. Gleichzeitig behindert ein fetaler Hyperinsulinismus die SurfactantBildung mit dem Risiko des Atemnotsyndroms beim Neugeborenen. Bei drohender Frühgeburt vor der 34. SSW ist daher eine fetale Lungenreifeinduktion mit $2 \times 12 \mathrm{mg}$ Betamethason im Abstand von 24 Stunden angezeigt. Nach Beginn der Glukokortikoidgabe muss für 4 Tage mit einer Steigerung des Insulinbedarfs um $20-40 \%$ gerechnet und die Insulindosis prospektiv angepasst werden.

Bei Glukokortikoidgabe zur Lungenreifeinduktion muss für 4 Tage mit einer Steigerung des Insulinbedarfs um 20-40\% gerechnet und die Dosis angepasst werden.

\section{Hypertonie, Präeklamsie/Eklampsie}

Die Präeklampsie ist definiert als ein Blutdruckanstieg $>140 / 90$ mmHg systolisch in Kombination mit einer Proteinausscheidung $>300 \mathrm{mg} / 24 \mathrm{~h}$ nach 20 SSW. Bei einer Gestationshypertonie fehlt die Proteinurie. Bei einer schweren Präeklampsie werden Blutdruckwerte $>170 \mathrm{mmHg}$ systolisch und/oder $>110 \mathrm{mmHg}$ diastolisch gemessen. Ziel der Intervention bei diesen Befunden ist die Vermeidung einer Eklampsie, die u.a. durch das Auftreten zerebraler Krampfanfälle und dem Risiko intrazerebraler Blutungen gekennzeichnet ist. Leitsymptom des HELLP-Syndroms, einer typischen Komplikation der Präeklampsie, ist der heftige rechtsseitige Oberbauchschmerz. Beendet wird der lebensbedrohliche Zustand meist nur durch eine schnelle Entbindung.

Durch Einnahme von $100 \mathrm{mg}$ Acetylsalicylsäure/Tag, beginnend vor der 12.SSW, lässt sich in Fällen, die als Hochrisiko durch die Geburtsmediziner eingestuft werden, das Präeklampsierisiko im Rahmen der Primärprävention vermindern. Hierzu zählen z. B. Schwangere mit Typ-1-Diabetes mit arterieller Hypertonie und Nephropathie. Nach 20 SSW soll eine Hochdrucktherapie ab $160 \mathrm{mmHg}$ systolisch oder $100 \mathrm{mmHg}$ diastolisch begonnen und eine stationäre Überwachung erfolgen. Das individuelle Präeklampsierisiko kann durch eine Doppler-Sonografie der Arteria uterina abgeschätzt werden (Frühdiastolische Widerstandserhöhung: Einkerbung der Flusskurve, sog. „Notch"). 
Das Präeklampsierisiko lässt sich bei HochrisikoPatientinnen durch die Einnahme von $100 \mathrm{mg}$

Acetylsalicylsäure vermindern.

\section{Kernaussagen}

- Der gute Ausgang einer Schwangerschaft bei bekanntem Typ-1- oder Typ-2-Diabetes verlangt eine bereits perikonzeptionell optimierte Stoffwechseleinstellung.

- Die Prävalenz des Gestationsdiabetes hat sich in Deutschland in den letzten 10 Jahren verdreifacht. Seit März 2012 ist das Screening auf Gestationsdiabetes Leistung der gesetzlichen Krankenkassen.

\section{Abstract}

In Germany the prevalence of pregnancies in women with type 1, type 2 and gestational diabetes is increasing. Diabetes care during pregnancy should be managed in close cooperation of diabetologists, obstetricians and midwives. In cases of preconceptional known diabetes good pregnancy outcome is associated with quality of periconceptional blood glucose control. Women with type 2 diabetes should be encouraged to reduce excess weight before pregnancy and replacing oral antidiabetic drugs or GLP-1 analogues with insulin. Every pregnant woman should be screenend on gestational diabetes between 24 and 28 weeks of pregnancy.
Aktualisierte Fassung des folgenden Beitrags: http://dx. doi.org/10.1055/s-2008-1077030

Interessenkonflikt: Die Autoren erklären, dass keine Interessenkonflikte bestehen.

\section{Über die Autoren}

\section{Helmut Kleinwechter}

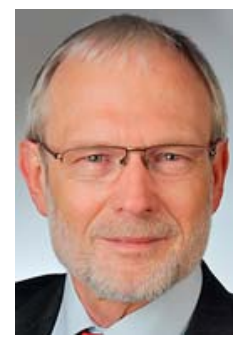

Dr. Helmut Kleinwechter arbeitet als Internist und Diabetologe seit 20 Jahren im „diabetologikum kiel“, einer von der Deutschen Diabetes-Gesellschaft zertifizierten Einrichtung zur Betreuung von Menschen mit Typ-1und Typ-2-Diabetes.

\section{Ute Schäfer-Graf}

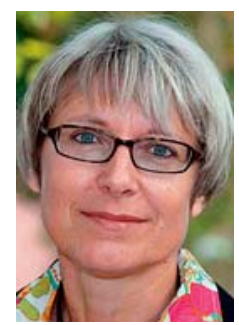

Prof. Dr. Ute Schäfer-Graf arbeitet seit 2008 als Oberärztin im Berliner Diabetes-Zentrum für Schwangere am St.Joseph-Krankenhaus. Sie ist Fachärztin für Gynäkologie und Geburtshilfe, Perinatologin und Diabetologin.

\section{Korrespondenzadresse}

Dr. med. Helmut Kleinwechter

diabetologikum kiel

Diabetes-Schwerpunktpraxis u. Schulungszentrum

Alter Markt 11

24103 Kiel

E-Mail: arzt@diabetologikum-kiel.de 


\section{Literatur}

1 Kleinwechter H, Bührer C, Hunger-Battefeld W et al. Diabetes und Schwangerschaft - Evidenzbasierte Leitlinien der Deutschen Diabetes-Geseellschaft.http://www.deutsche-diabetesgesellschaft.de/fileadmin/Redakteur/Leitlinien/Evidenzbasierte_Leitlinien/EBL_Schwangerschaft_2008.pdf Zugriff 11.2.2013

2 Kleinwechter H, Schäfer-Graf U, Bührer C et al. Gestationsdiabetes mellitus (GDM) - Evidenzbasierte Leitlinie zu Diagnostik, Therapie und Nachsorge der Deutschen Diabetes-Gesellschaft und der Deutschen Gesellschaft für Gynäkologie und Geburtshilfe.http://www.deutsche-diabetes-gesellschaft.de/ fileadmin/Redakteur/Leitlinien/Evidenzbasierte_Leitlinien/ Gestationsdiabetes_EbLL_Endfassung_2011_08_11_.pdf Zugriff 16.1.2013

3 Kleinwechter H, Schäfer-Graf U, Bührer C et al. Diabetes und Schwangerschaft. Diabetologie 2012; 7 (Suppl. 2): S185S 191

4 Kleinwechter H, Schäfer-Graf U, Bührer C et al. Gestationsdiabetes mellitus (GDM) - Diagnostik, Therapie u. Nachsorge. Praxisleitlinie der Deutschen Diabetes-Gesellschaft und der Deutschen Gesellschaft für Gynäkologie und Geburtshilfe. http://www.deutsche-diabetes-gesellschaft.de/fileadmin/ Redakteur/Leitlinien/Praxisleitlinien/2012/DuS_S2-12_Praxisempfehlungen_Kleinwechter-etal_GDM_S174-184.pdf Zugriff 16.1.2013

5 Kleinwechter H, Bührer C, Hunger-Battefeld W et al. Diabetes und Schwangerschaft - Patientenversion der Leitlinie der Deutschen Diabets-Gesellschaft.http://www.deutsche-diabetes-gesellschaft.de/fileadmin/Redakteur/Leitlinien/Patientenleitlinien/PatL_Schwangerschaft_2008.pdf Zugriff 16.1.2013

6 Kleinwechter $\mathrm{H}$, Schäfer-Graf U, Bührer C et al. Schwangerschaftsdiabetes (Gestationsdiabetes) - Leitlinie für Patientinnen, Schwangere und Interessierte zu Diagnostik, Behandlung u. Nachsorge der Deutschen Diabetes-Gesellschaft und der Deutschen Gesellschaft für Gynäkologie und Geburthilfe. http://www.deutsche-diabetes-gesellschaft.de/fileadmin/
Redakteur/Leitlinien/Patientenleitlinien/GDM_Patienten_LL_END_2012_04_17.pdf Zugriff 16.1.2013

7 Schäfer-Graf U. Kontrazeption bei Frauen mit Diabetes. Diabetologe 2012; 8: 321-331

8 Mathiesen E, Hod M, Ivanisevic M et al. Maternal efficacy and safety outcomes in a randomized, controlled trial comparing insulin detemir with NPH insulin in 310 pregnant women with type 1 diabetes. Diabetes Care 2012; 35: 2012-2017

9 Ringholm L, Pedersen-Bjergaard U, Thorsteinsson B et al. Hypoglycemia during pregnancy in women with Type 1 diabetes. Diabet Med 2012; 29: $558-566$

10 Chico M, Levine S, Lewis D. Normoglycemic diabetic ketoacidosis in pregnancy. J Perinatol 2008; 28: 310-312

11 Bullo M, Tschumi S, Bucher B et al. Pregnancy Outcome Following Exposure to Angiotensin-Converting Enzyme Inhibitors or Angiotensin Receptor Antagonists. A systematic review. Hypertension 2012; 60: $444-450$

12 Nielsen R, Müller C, Damm P et al. Reduced prevalence of early delivery in women with Type 1 diabetes and microalbuminuria - possible effect of early antihypertensive treatment during pregnancy. Diab Med 2006; 23: 426-431

13 Ásbjörnsdóttir B, Rasmussen S, Kelstrup L et al. Impact of restricted maternal weight gain on fetal growth and perinatal morbidity in obese women with type 2 diabetes. Diabetes Care 2012: 10.337/dc12-1232

14 The HAPO Study Cooperative Research Group. Hyperglycemia and adverse pregnancy outcomes. N Engl J Med 2008; 358: $1991-2002$

15 Schaefer-Graf U, Kjos S, Fauzan O et al. Randomized trial evaluating a predominantely fetal growth-based strategy to guide management of gestational diabetes in Caucasian women. Diabetes Care 2004; 27: 297-302

16 Löbner K, Knopff A, Baumgarten A et al. Predictors of postpartum diabetes in women with gestational diabetes mellitus. Diabetes 2006; 55: $792-797$ 


\section{CME-Fragen}

\section{CME.thieme.de}

\section{CME-Teilnahme}

- Viel Erfolg bei Ihrer CME-Teilnahme unter http://cme.thieme.de

- Diese Fortbildungseinheit ist 12 Monate online für eine CME-Teilnahme verfügbar.

- Sollten Sie Fragen zur Online-Teilnahme haben, unter http://cme.thieme.de/hilfe finden Sie eine ausführliche Anleitung.

1 Das Risiko, an einem Typ-1-Diabetes zu erkranken, liegt für die Tochter einer Mutter mit Typ-1-Diabetes bei ca....
A $11 \%$ bis zu einem Alter von 2 Jahren
B $0,3 \%$ bis zu einem Alter von 25 Jahren
C $12 \%$ bis zu einem Alter von 5 Jahren
D $20 \%$ bis zu einem Alter von 10 Jahren
E $5,3 \%$ bis zu einem Alter von 20 Jahren

2 Über welche Risiken muss eine Patientin mit Typ-1-Diabetes mellitus bei Schwangerschaftsplanung nicht informiert werden?
A erhöhtes Fehlbildungsrisiko
B erhöhtes Risiko für Trisomie 21
C erhöhtes Risiko für Präeklampsie
D erhöhtes Risiko für Frühgeburten
E erhöhte Verlegungsrate des Neugeborenen in die Kinderklinik

3 Welches Insulin wird für Schwangere mit manifestem Diabetes nicht empfohlen?
A Glargin
B NPH-Insulin
C Detemir
D Aspart
E Kurzwirksames Humaninsulin

4 Was unterscheidet eine schwangere Patientin mit Typ-2-Diabetes typischerweise von einer Patientin mit Typ-1-Diabetes?
A geringeres Körpergewicht
B bessere präkonzeptionelle Folsäureeinnahme
C engmaschigere diabetologische Kontrollen
D kürzere Diabetesdauer
E höhere Erkrankungsrate an Retinopathie

Welche Aussage ist falsch? Die Blutglukose einer Schwangeren mit Typ-2-Diabetes kann ansteigen durch ...
A körperliche Anstrengung
B Bettruhe
C Lungenreifeinduktion mit Betamethason
D Tokolyse mit Fenoterol
E fieberhafte Pyelonephritis 


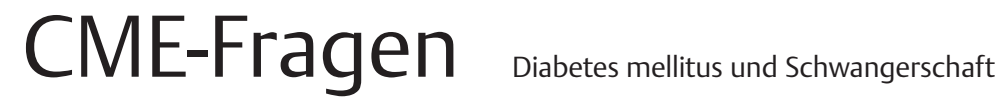

6 Im Jahr 2011 betrug die Häufigkeit des bei der Geburt von der Perinatalstatistik erfassten Gestationsdiabetes in Deutschland ...
A $0,3 \%$
B $1,3 \%$
C $4,4 \%$
D $8,8 \%$
E $16,1 \%$

7 Die Diagnose eines Gestationsdiabetes wird gesichert durch...
A die Messung des HbA1c
B typische klinische Symptome
C den Nachweis von Uringlukose 3+
D einen auffälligen Ultraschallbefund des Fetus
E einen $75 \mathrm{~g}$-oGTT

8 Ein Gestationsdiabetes liegt vor, wenn ...
A bei einer asymptomatischen Schwangeren mit 14 SSW einmalig eine Gelegenheitsglukose von $230 \mathrm{mg} / \mathrm{dl}(12,8 \mathrm{mmol} / \mathrm{l})$ gemessen wird
B der HbA1c in der 7.SSW 6,7\% beträgt
C nach $75 \mathrm{~g}$ Glukosebelastung der 2-Stundenwert $150 \mathrm{mg} / \mathrm{dl}(8,3 \mathrm{mmol} / \mathrm{l})$ beträgt
D mit 26+3 SSW im 75-g-oGTT ein Nüchternwert von $95 \mathrm{mg} / \mathrm{dl}(5,3 \mathrm{mmol})$ gemessen wird
E im 50-g-Screening-Test mit $27+3$ SSW ein Wert von $200 \mathrm{mg} / \mathrm{dl}(11,1 \mathrm{mmol} / \mathrm{l})$ vorliegt

9 Welches Medikament wird zur Pharmakotherapie des GDM empfohlen?
A Metformin
B Liraglutid
C NPH-Insulin
D Glimepirid
E Dapagliflozin

10 Welches Medikament ist zur oralen Behandlung des Hochdrucks in der Schwangerschaft am besten geeignet?
A Alpha-Methyl-Dopa
B Candesartan
C Enalapril
D Atenolol
E Dihydralazin 